\title{
DESEMPEÑO PRODUCTIVO DEL CAPAZ (Pimelodus grosskopfii, Steindachner, 1879) BAJO DIFERENTES DENSIDADES DE SIEMBRA Y DE CONTENIDO DE PROTEINA EN LA DIETA
}

\author{
PRODUCTIVE PERFORMANCE OF CAPAZ (Pimelodus grosskopfii, Steindachner, 1879) \\ UNDER DIFFERENT STOCKING DENSITIES AND PROTEIN CONTENT IN DIET
}

Silvia Carrera y Rubén Darío Valbuena-Villareal

\begin{abstract}
RESUMEN
El capaz es un pez representante de la familia Pimelodidae, cuya producción proviene de la pesquería en el río Magdalena y en la represa de Betania. Aunque es una especie de interés comercial, los estudios sobre su cultivo aún se desconocen. Con el objetivo de evaluar el desempeño productivo del capaz bajo diferentes densidades de siembra y con diferentes niveles de proteína en la dieta, se utilizaron 450 alevinos con un peso promedio de $37,6 \pm 2,4 \mathrm{~g}$ y una talla inicial de 17,5 $\pm 1,7 \mathrm{~cm}$, previamente acondicionados a consumo de concentrado comercial y durante un periodo productivo de 6 meses en jaulas flotantes en el embalse de Betania. Los animales fueron distribuidos en dos densidades de siembra $\left(25 \mathrm{y} 50\right.$ peces $\left./ \mathrm{m}^{3}\right)$ y alimentados con dos diferentes niveles de Proteína Bruta (PB) en la dieta (25 y 32 \%). Se obtuvieron mejores resultados para la densidad de siembra de 25 peces $/ \mathrm{m}^{3}$ y alimentados con concentrado de $25 \%$ de proteína, con los valores más altos en cuanto a ganancia de peso (150 $\pm 4 \mathrm{~g})$, tasa de crecimiento especifico $(0,8 \pm 0,002)$ y factor de crecimiento relativo $(7,39 \pm 0,06)$. De acuerdo con los resultados obtenidos se puede concluir que la densidad de 25 peces $/ \mathrm{m}^{3}$ y el alimento con un contenido de $25 \%$ de proteína presentan las mejores condiciones para el cultivo comercial de Pimelodus grosskopfii en jaulas flotantes.
\end{abstract}

PALABRAS CLAVE: Pimelodus grosskopfii, desempeño productivo, densidad de siembra

\begin{abstract}
The capaz is a representative of the family Pimelodidae fish, whose production comes from fishing in Magdalena River and the Embalse de Betania. Although a species of commercial interest, studies on its cultivation are scarce. In order to evaluate productive performance of capaz under different densities and different levels of protein in the diet, were used 450 fingerlings with an average weight of $37.6 \pm 2.4 \mathrm{~g}$ and an initial size of $17.5 \pm 1.7 \mathrm{~cm}$ preconditioned for consumption of commercial concentrate and during a productive period of six months in floating cages. The animals were divided into 2 densities ( 25 and 50 fish $/ \mathrm{m}^{3}$ ) and fed 2 different protein levels Gross (PB) in the diet (25 and $32 \%$ ). Best results were obtained for planting density of 25 fish $/ \mathrm{m}^{3}$ and concentrate fed $25 \%$ protein, with the highest values in terms of weight gain (150 $\left.\pm 4 \mathrm{~g}\right)$, specific growth rate $(0.8 \pm 0.002)$ and relative growth factor $(7.39 \pm 0.06)$. According to the results obtained it can be concluded that the density of 25 fish / $\mathrm{m}^{3}$ and food with a content of $25 \%$ protein present the best conditions for the commercial cultivation of Pimelodus grosskopfii in floating cages.
\end{abstract}

KEY WORDS: Pimelodus grosskopfii, performance assessment, stocking density

\section{INTRODUCCIÓN}

En los últimos años la acuicultura se ha convertido en uno de los sectores de la economía con un rápido crecimiento, este auge se ha centrado en unas cuantas 20 especies, las cuales en su mayoría son foráneas (CruzCasallas et al., 2010). La introducción de nuevas especies, preferiblemente nativas, a los sistemas de producción puede llegar a ser una alternativa para la diversificación de la acuicultura y adicionalmente una posible solución que merme el impacto que genera la pesca de extracción sobre los recursos pesqueros (Petrere et al., 2004). Esta diversificación implica el estudio de algunos aspectos básicos como el cultivo y

Dirección de los autores:

Corporación Centro de Desarrollo Piscícola Surcolombiano-Acuapez, Calle 23 \# 5a -75, Barrio Sevilla, Teléfono y fax: 8721468, Colombia. E-mail: productividadacuapez@gmail.com (S.C). Universidad Surcolombiana, Avenida Pastrana Borrero, Teléfono 8754753. Neiva-Huila, Colombia (R.D.V.V). 
ceba de especies con potencial para ser cultivadas, de tal forma que permitan establecer paquetes tecnológicos para optimizar la producción a escala comercial (Linhart et al., 2002).

Los Siluriformes tienen una amplia distribución a nivel mundial, principalmente en aguas continentales; son considerados el grupo de peces más representativos, con más de 30 familias, de la fauna suramericana, (RománValencia, 2001). Para este grupo de peces, existen estudios en los que se han evaluado el desempeño productivo de algunas especies tales como el yaque, Leiarius marmoratus (Cruz-Casallas et al., 2010; MurilloPacheco et al., 2012), bagre de canal, Ictalurus punctatus (Sena de Souza et al., 2005) y Clarias gariepinus (Amisah et al., 2009).

El capaz (Pimelodus grosskopfii) es una especie representante de la familia Pimelodidae, que habita en las cuencas de los ríos Magdalena, Cauca, San Jorge, Sinú, Cesar, Atrato, Baudó y Catatumbo (Villa-Navarro, 2002) y los embalses de Betania (Huila) y Prado (Tolima). Es una especie omnívora con preferencia por insectos, macroinvertebrados y larvas de peces (Villa-Navarro y Losada-Prada, 1999). Por la calidad de su carne tiene una alta aceptación a nivel comercial, lo cual la hace una especie interesante para producir comercialmente.

Las investigaciones de Pimelodus grosskopfii en Colombia, se han desarrollado en torno a evaluaciones sobre aspectos generales de su biología (Cala et al., 1996; Cala, 1997; Valbuena-Villarreal et al., 2012a), experimentos de reproducción inducida (ValbuenaVillarreal et al., 2010) y sobre la primera alimentación (Valbuena-Villarreal et al., 2012b; Valbuena-Villarreal et al., 2013). No obstante, su rendimiento bajo determinadas condiciones de cultivo aún se desconoce. Por lo tanto, el objetivo de este trabajo fue evaluar el desempeño productivo del capaz en jaulas flotantes en el embalse de Betania, bajo diferentes densidades de siembra y a diferentes niveles de proteína bruta en la dieta.

\section{MATERIALES Y MÉTODOS}

\section{Área de estudio}

El experimento se llevó a cabo en la estación piscícola de Comepez, ubicada en la ensenada las Barcazas del embalse de Betania, a $5 \mathrm{~km}$ del Puerto de Seboruco. El embalse se ubica al sur-occidente del departamento de
Huila, a $35 \mathrm{~km}$ de la ciudad de Neiva a una altura de 557 $\mathrm{m}$, en la cuenca alta del río Magdalena, con un volumen de 2000 millones de $\mathrm{m}^{3} \mathrm{y}$ un flujo de $473 \mathrm{~m}^{3} / \mathrm{s}$, formado por el represamiento de los ríos Yaguara y Magdalena (Mancera-Rodríguez y Cala, 1997).

\section{Material biológico}

Se utilizaron 450 peces con un peso inicial promedio de $37,6 \pm 2,4 \mathrm{~g}$ y una talla de $17,5 \pm 1,7 \mathrm{~cm}$. Los alevinos fueron obtenidos de la Estación Piscícola de la AUNAP, ubicada en el municipio de Gigante, Huila. Los ejemplares provenían de un desove obtenido por reproducción artificial, inducida con extracto de hipófisis de Carpa (EHC), siguiendo el protocolo propuesto por Valbuena-Villarreal et al. (2010).

\section{Diseño experimental}

Para el desarrollo del experimento se usaron jaulas de $1 \mathrm{~m}^{3}$, que contaban con un sistema de flotación. Para la construcción de las jaulas se utilizaron varillas de una pulgada y mallas plásticas de nylon y de color negro. Se planteó un diseño experimental multifactorial completamente aleatorizado, el cual consistió en evaluar dos densidades de siembra (25 y 50 peces $/ \mathrm{m}^{3}$ ) y dos niveles de Proteína Bruta (PB) de un concentrado comercial ( 25 y $32 \%$ ) con tres repeticiones cada uno. Los ensayos tuvieron una duración de 180 días comprendidos entre los meses de febrero y agosto de 2014.

Una vez distribuidos los tratamientos en la estación piscícola, los peces fueron sometidos a tres días de aclimatación, al final de los cuales se dio inicio a la alimentación. La alimentación fue suministrada en dos raciones al día (9:00 y 15:00 horas). La cantidad de alimento suministrada fue ajustada de acuerdo con la biomasa determinada en los muestreos periódicos, teniendo en cuenta los criterios utilizados por Mojica et al. (2003), así: hasta $30 \mathrm{~g}$ a saciedad, entre 30 y $150 \mathrm{~g}$ el $3,5 \%$ de la biomasa total, entre 150 y 380 g el $2,5 \%$ de la biomasa total y a partir de los $400 \mathrm{~g}$ de peso corporal el $1 \%$ de la biomasa total.

Semanalmente se midieron los parámetros de calidad del agua en cada tratamiento, se registraron valores de temperatura $\left({ }^{\circ} \mathrm{C}\right)$, oxígeno disuelto $(\mathrm{mg} / \mathrm{L}), \mathrm{pH}$, concentración de nitritos (mg/L), amonio (mg/L), alcalinidad (mg/L) y dureza (mg/L). Para las mediciones se empleó una sonda multiparamétrica (YSI 566) y un kit colorimétrico de aguas (Aquamerck). 


\section{Evaluación del desempeño productivo}

Para determinar el desempeño productivo se realizaron muestreos quincenales del $10 \%$ de la población, se registraron las siguientes variables: longitud total $(\mathrm{cm})$, longitud estándar $(\mathrm{cm})$ con un ictiómetro y peso (g) con una balanza digital (Ohaus Scout Pro). Al final de los seis meses se realizó la cosecha y se calcularon las variables productivas: 1 . Ganancia de peso (GP) = peso final (g) - peso inicial (g), 2. Ganancia de talla (GT): talla final $(\mathrm{cm})$ - talla inicial $(\mathrm{cm}), 3$. Ganancia diaria de peso $(\mathrm{GDP})(\mathrm{g} /$ día $)=$ peso final - peso inicial/días del ciclo de producción, 4. Conversión alimenticia (TCA) = consumo de alimento (g)/ganancia de peso total (g), 5. Factor de crecimiento relativo $(\mathrm{FCR})=$ peso final (g)/ longitud total final (cm), 6. Factor de condición $(\mathrm{Kn})=$ peso final $(\mathrm{g}) /$ longitud total $(\mathrm{cm})^{3}, 7$. Tasa de crecimiento especifico (TCE) $=$ Ln peso final $/$ Ln peso inicial/ tiempo de cultivo x 100 y 8 . Sobrevivencia (\%): ( $\mathrm{n}^{\circ}$ final de individuos $/ \mathrm{n}^{\circ}$ inicial de individuos) x 100 .

Adicionalmente, al momento de la cosecha, se realizó el sacrificio al $10 \%$ de la población, se hicieron disecciones y se pesó el hígado (g), el intestino (g) y la grasa visceral (g), con el propósito de calcular los siguientes índices: índice viscerosomático (IVS): 100* (peso de vísceras / peso corporal), índice hepatosomático (IHS): 100* (peso de hígado /peso corporal) e índice de grasa visceral (IGV): 100* (peso de grasa en la vísceras / peso corporal).

\section{Análisis estadístico}

Inicialmente para describir los datos se usaron estadísticos de tendencia central, tales como la media \pm desviación estándar. Posteriormente, se analizaron los efectos de los tratamientos sobre la producción, para lo cual se realizó un análisis de varianza (ANOVA) de dos vías, previo a este análisis se verificaron los supuestos de normalidad (prueba de Kolmogorov-Smirnov) y homogeneidad de varianzas (prueba de Levene). Cuando se presentaron diferencias estadísticamente significativas, se compararon las medias entre los diferentes tratamientos con la prueba a posteriori de Tukey. El criterio de significancia fue $\mathrm{p}<0,05$. Los análisis estadísticos fueron realizados usando el programa SPSS v20.

\section{RESULTADOS}

Las variables físico-químicas del agua en todos los tratamientos no presentaron diferencias estadísticamente significativas (Tabla 1). La temperatura se mantuvo entre 26,5 y $27,3{ }^{\circ} \mathrm{C}$, el oxígeno disuelto entre 3,2 y $3,6 \mathrm{mg} / \mathrm{L}$ y el pH entre 7,7 y 7,8 .

Se registraron valores máximos de talla $(26,1 \pm 0,9$ $\mathrm{cm})$ y de peso $(166 \pm 306 \mathrm{~g})$, en los tratamientos que incluyeron cultivos a una densidad de 25 peces/ $\mathrm{m}^{3} \mathrm{y}$ alimentados con una dieta de $25 \%$ de proteína bruta (PB, Figura 1). La densidad de siembra no tuvo un efecto sobre la ganancia de peso de los ejemplares $(p=0,0606)$, mientras que el nivel de proteína en la dieta sí $(p=0,0285)$, de igual manera se mostró una interacción significativa entre ambos factores $(p=0,0126)$. Con relación a la talla, ni la densidad de siembra ( $p=0,0633)$, ni el nivel de proteína bruta en la dieta ( $p=0,3088$ ) mostraron un efecto estadísticamente significativo sobre la ganancia de peso.

Tabla 1. Parámetros físicos y químicos del agua.

\begin{tabular}{|c|c|c|c|c|c|c|c|c|}
\hline \multicolumn{2}{|c|}{ Tratamientos } & \multirow[b]{2}{*}{$\begin{array}{c}\text { Temperatura } \\
\left({ }^{\circ} \mathrm{C}\right)\end{array}$} & \multirow{2}{*}{$\begin{array}{c}\text { Oxígeno } \\
\text { disuelto } \\
(\mathrm{mg} / \mathrm{L})\end{array}$} & \multirow[b]{2}{*}{$\mathrm{pH}$} & \multirow[b]{2}{*}{$\begin{array}{l}\text { Amonio } \\
(\mathrm{mg} / \mathrm{L})\end{array}$} & \multirow[b]{2}{*}{$\begin{array}{c}\text { Nitritos } \\
\text { (mg/L) }\end{array}$} & \multirow[b]{2}{*}{$\begin{array}{c}\text { Alcalinidad } \\
\text { (mg/L) }\end{array}$} & \multirow[b]{2}{*}{$\begin{array}{l}\text { Dureza } \\
\text { (mg/L) }\end{array}$} \\
\hline $\begin{array}{c}\text { Densidad } \\
\left(\text { peces/m } / \mathrm{m}^{3}\right)\end{array}$ & $\begin{array}{l}\text { Proteína } \\
(\%)\end{array}$ & & & & & & & \\
\hline 25 & 25 & $27,3 \pm 1,3$ & $3,5 \pm 0,7$ & $7,7 \pm 0,3$ & $0,2 \pm 0,01$ & $0,007 \pm 0,001$ & $50 \pm 1$ & $46 \pm 2$ \\
\hline 50 & 25 & $26,8 \pm 1,2$ & $3,6 \pm 0,5$ & $7,8 \pm 0,3$ & $0,4 \pm 0,01$ & $0,019 \pm 0,001$ & $48 \pm 2$ & $53 \pm 1$ \\
\hline 25 & 32 & $27,1 \pm 0,5$ & $3,2 \pm 0,2$ & $7,8 \pm 0,2$ & $0,3 \pm 0,02$ & $0,018 \pm 0,001$ & $50 \pm 1$ & $56 \pm 1$ \\
\hline 50 & 32 & $26,5 \pm 0,5$ & $3,2 \pm 0,5$ & $7,8 \pm 0,3$ & $0,4 \pm 0,02$ & $0,019 \pm 0,001$ & $49 \pm 1$ & $53 \pm 1$ \\
\hline
\end{tabular}




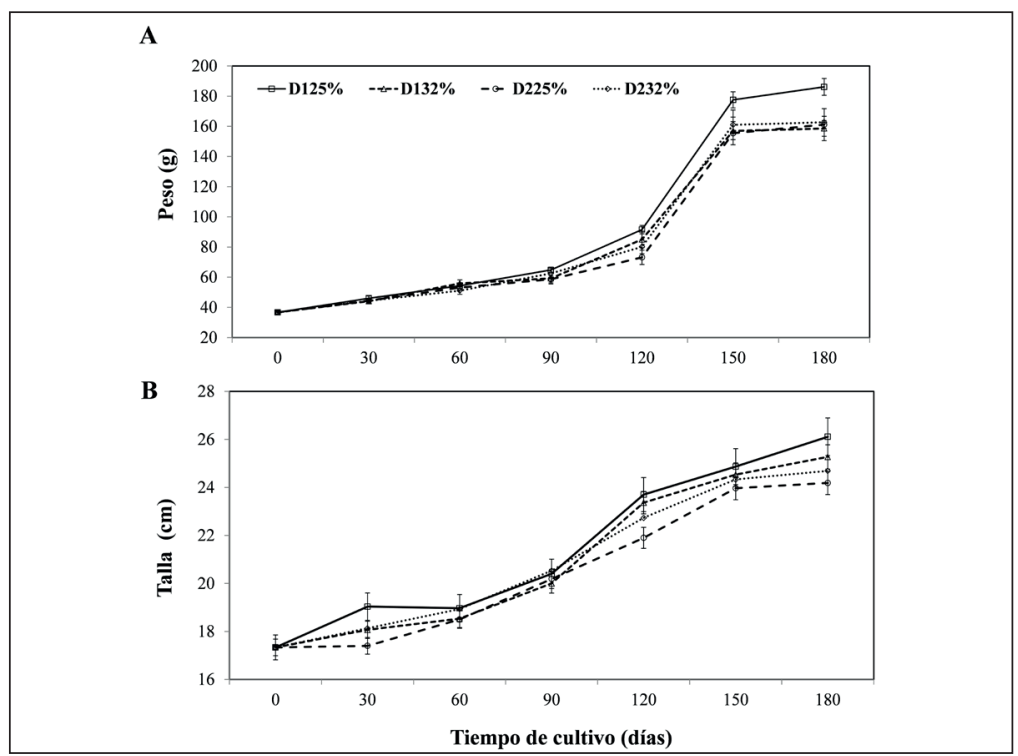

Figura 1. A) Peso y B) Talla del capaz Pimelodus grosskopfii cultivado en jaulas flotantes.
En cuanto al desempeño productivo, se presentaron mejores resultados en ganancia diaria de peso $(0,82 \pm 0,02$ $\mathrm{g} /$ día), tasa de crecimiento específica $(0,8 \pm 0,002 \%)$ y factor de crecimiento relativo $(7,39 \pm 0,06)$ en los tratamientos con cultivo a densidad de 25 peces $/ \mathrm{m}^{3}$ y alimentados con el $25 \%$ de proteína. Las mejores tasas de conversión alimenticia se obtuvieron en los animales cultivados a una densidad de 25 peces/ $\mathrm{m}^{3}$ y alimentados con 25 y $32 \%$ de proteína y en densidades de 50 peces/ $\mathrm{m}^{3}$ y alimentados con $32 \%$ de proteína. La mejor sobrevivencia ( $80 \pm 2 \%$ ), se obtuvo con la densidad 50 peces $/ \mathrm{m}^{3} \mathrm{y}$ alimentados con $32 \%$ y $25 \%$ de PB y el factor de condición (Kn) fue similar en todos los tratamientos (Tabla 2).

Tabla 2. Desempeño productivo del capaz Pimelodus grosskopfii.

\begin{tabular}{|c|c|c|c|c|}
\hline \multirow{2}{*}{ Variables } & \multicolumn{2}{|c|}{ Concentrado con $25 \%$ de PB } & \multicolumn{2}{|c|}{ Concentrado con $32 \%$ de PB } \\
\hline & 25 peces $/ \mathrm{m}^{3}$ & 50 peces $/ \mathrm{m}^{3}$ & 25 peces $/ \mathrm{m}^{3}$ & 50 peces $/ \mathrm{m}^{3}$ \\
\hline Ganancia de peso (g) & $150 \pm 4^{a}$ & $125 \pm 3^{b}$ & $122 \pm 9^{b}$ & $126 \pm 13^{b}$ \\
\hline Ganancia de talla (cm) & $7,9 \pm 0,3^{\text {a }}$ & $6,8 \pm 0,9^{a}$ & $8,2 \pm 0,9^{a}$ & $7,4 \pm 0,7^{a}$ \\
\hline Ganancia diaria de peso GDP (g/día) & $0,82 \pm 0,02 a^{a}$ & $0,69 \pm 0,01^{b}$ & $0,67 \pm 0,05^{b}$ & $0,69 \pm 0,07^{b}$ \\
\hline Conversión alimenticia (TCA) & $2,1 \pm 0,24^{a}$ & $2,6 \pm 0,3^{b}$ & $2,1 \pm 0,24^{a}$ & $1,9 \pm 0,2^{a}$ \\
\hline Tasa de crecimiento especifica TCE & $0,8 \pm 0,002^{a}$ & $0,78 \pm 0,002^{b}$ & $0,78 \pm 0,008^{b}$ & $0,78 \pm 0,012^{b}$ \\
\hline Factor de crecimiento relativo FCR & $7,39 \pm 0,06^{a}$ & $6,23 \pm 0,30 \mathrm{ab}$ & $6,69 \pm 0,34^{b}$ & $6,6 \pm 0,73 a b$ \\
\hline Sobrevivencia (\%) & $70 \pm 2^{c}$ & $80 \pm 2^{a}$ & $72 \pm 2^{b c}$ & $77 \pm 2^{a b}$ \\
\hline Factor de Condición (Kn) & $0,011 \pm 0,0001^{\mathrm{a}}$ & $0,009 \pm 0,0011^{a}$ & $0,011 \pm 0,0011^{\mathrm{a}}$ & $0,108 \pm 0,0018^{a}$ \\
\hline
\end{tabular}

"Los tratamientos con las mismas letras pertenecen a un mismo grupo homogéneo identificado por la prueba de rangos múltiples de Tukey. Letras diferentes indican diferencias significativas entre tratamientos $(\mathrm{P}<0,05)$.

En cuanto a los índices corporales obtenidos al final del ensayo, se mostraron diferencias significativas entre los índices viscerosomático (IVS) y de grasa visceral (IGV). Los mayores valores se registraron en los animales cultivados densidades de 25 peces $/ \mathrm{m}^{3} \mathrm{y}$ alimentados con concentrado con $32 \%$ de PB $(9,83 \pm 0,77)$ y en la densidad de 25 peces $/ \mathrm{m}^{3}$ y alimentados con 32 y $25 \%$ de PB $(5,16 \pm 0,44$ y 4,34 $\pm 0,76)$, respectivamente, a diferencia del índice hepatosomático que fue similar en todos los tratamientos (Tabla 3). 
Tabla 3. Índices corporales del capaz Pimelodus grosskopfii.

\begin{tabular}{lcccr}
\hline \multirow{2}{*}{ Variables } & \multicolumn{2}{c}{ Concentrado con $\mathbf{2 5} \%$ de PB } & \multicolumn{2}{c}{ Concentrado con 32 \% de PB } \\
\cline { 2 - 5 } & $\mathbf{2 5} \mathbf{p e c e s} / \mathbf{m}^{\mathbf{3}}$ & $\mathbf{5 0}$ peces $/ \mathbf{m}^{\mathbf{3}}$ & $\mathbf{2 5} \mathbf{~ p e c e s} / \mathbf{m}^{\mathbf{3}}$ & $\mathbf{5 0} \mathbf{p e c e s} / \mathbf{m}^{\mathbf{3}}$ \\
\hline Índice viscerosomático IVS (\%) & $8,54 \pm 0,56^{\mathrm{b}}$ & $7,64 \pm 0,15^{\mathrm{bc}}$ & $9,83 \pm 0,77^{\mathrm{a}}$ & $7,37 \pm 0,22^{\mathrm{c}}$ \\
Índice hepatosomático IHS (\%) & $1,27 \pm 0,05^{\mathrm{a}}$ & $1,19 \pm 0,06^{\mathrm{a}}$ & $1,00 \pm 0,09^{\mathrm{a}}$ & $1,20 \pm 0,42^{\mathrm{a}}$ \\
Índice de grasa visceral IGV (\%) & $4,34 \pm 0,76^{\mathrm{ab}}$ & $3,67 \pm 0,41^{\mathrm{bc}}$ & $5,16 \pm 0,44^{\mathrm{a}}$ & $3,07 \pm 0,29^{\mathrm{c}}$ \\
\hline
\end{tabular}

Los tratamientos con las mismas letras pertenecen a un mismo grupo homogéneo identificado por la prueba de rangos múltiples de Tukey. Letras diferentes indican diferencias significativas entre tratamientos $(P<0,05)$.

\section{DISCUSIÓN}

En la producción comercial de organismos acuáticos, los parámetros de calidad del agua juegan un papel importante para optimizar su desempeño. El capaz es una especie que se encuentra en las cuencas alta y media del río Magdalena principalmente y al final del embalse de Betania sobre el Magdalena, estos sectores presentan valores de $\mathrm{pH}$ de 7 , temperatura promedio de $24,2{ }^{\circ} \mathrm{C}$ y una concentración de oxígeno de 5,9 mg/L (Cala et al., 1996). Por lo tanto, los parámetros medidos durante el experimento indican que esta especie puede ser cultivada comercialmente en este tipo de ecosistemas.

El efecto de la densidad de siembra sobre algunas variables productivas, se ha estudiado en algunas especies de silúridos de importancia comercial (Segura et al., 2004; Barcellos et al., 2004; Núñez et al., 2008). Con respecto al capaz, Kennedy-Luz y Zaniboni-Filho (2002), evaluaron la sobrevivencia de Pimelodus maculatus sobre tres densidades de siembra encontrando que a la menor densidad se obtuvo la mejor sobrevivencia. Por su parte Esquivel et al. (2008), evaluaron el aumento de talla y peso del bagre de canal, Ictalurus punctatus en cuatro densidades de siembra: $10,15,20$, y 25 peces/ $\mathrm{m}^{2}$; ellos encontraron que los mejores resultados se dan a densidades de 10 peces $/ \mathrm{m}^{2}$. Aunque la densidad de cultivo tiene una estrecha relación con la competencia por el recurso alimentario, no se encontraron diferencias significativas entre las densidades evaluadas sobre el peso y la talla. Estos resultados son consistentes con los encontrados en otras especies, por ejemplo para el pacú Piaractus mesopotamicus (Wicki y Luchini, 2005)

24 y para la perca plateada Bidyanus bidyanus (Rowland et al., 2006). Esto puede estar relacionado con la disponibilidad de nutrientes alóctonos que el embalse puede ofrecer a los organismos y que en este caso se presentó de manera similar en todos los tratamientos.
El efecto del alimento en el crecimiento de los peces depende de algunos factores como la especie, la talla y la edad del animal, su condición fisiológica y la calidad del agua (Phillips et al., 1998). En este estudio los mejores resultados relacionados con el peso se presentaron en la dieta que contenía $25 \%$ de proteína, contrario a los resultados encontrados en otras especies como los bagres: Ictalurus balsanus (Arce y Luna-Figueroa, 2003), Ictalurus punctatus (Cho y Lovell, 2001), Clarias isheriensis (Fagbenro y Arowosoge, 1991). Por otro lado no se encontraron diferencias significativas entre las talla vs dieta, resultados que se ha registrado en otras especies, tales como Rhamdia quelem (Coldebella et al., 2012). Teniendo en cuenta que la cantidad y calidad de proteína en la dieta es determinante en el crecimiento de los peces (Murillo-Pacheco et al., 2012), los resultados encontrados en este estudio sugieren que en esta especie, la proteína cumple un papel más importante en estado larval (Valbuena-Villareal et al., 2013) que en la etapa de alevinos.

El factor de condición, expresa el grado de bienestar de un organismo con relación al medio en que vive y está relacionado con la tasa de crecimiento y el nivel de confort de los animales (Namrata y Shamrao, 2013), basándose en la hipótesis de que los peces de mayor tamaño, a una determinada longitud, presentan una mejor condición (Froese, 2006). En este estudio, los valores obtenidos fueron inferiores a 1 en todos los tratamientos, resultados similares fueron encontrados en otros estudios (Cruz-Casallas et al., 2010). Lo que hace suponer que las condiciones dadas en todos los tratamientos provocaron un ambiente adecuado o de confort para el cultivo de esta especie.

Las mejores tasas de conversión alimenticia se obtuvieron en los animales cultivados a 25 peces $/ \mathrm{m}^{3}$ y alimentados con concentrado comercial de 32 y $25 \%$ 
de proteína y a 50 peces $/ \mathrm{m}^{3}$ alimentados con $32 \%$ de proteína. Valores similares se obtuvieron en el cultivo de otros silúridos como el yaque (Cruz-Casallas et al., 2010). Por lo tanto, estos resultados sugieren que la tasa de conversión alimenticia en una especie omnívora como el capaz, cultivada a altas densidades, podría estar ligada a la variedad de alimentos que se pueden encontrar en un sistema como los embalses.

Los índices corporales tales como el viscerosomático (IVS), hepatosomático (IHS) y de grasa visceral (IGV) están relacionados directamente con el factor de condición e inversamente con el grado de madurez del individuo (Cruz-Casallas et al., 2010), e igualmente tienden a reflejar procesos de almacenamiento y de transferencia de proteínas y lípidos para soportar procesos como la maduración y desove durante un determinado tiempo (González y Oyarzún, 2002). En este ensayo los individuos utilizados alcanzaron una edad de seis meses y presentaron índices hepatosomáticos relativamente bajos comparados con otras especies de silúridos, contrario a lo ocurrido con los índices viscerosomático y de grasa visceral (CruzCasallas et al., 2010), lo que indica posiblemente que en estado de reposo los animales tienden a almacenar grasa como mecanismo de reserva para su reproducción y/o posiblemente los animales tuvieron una sobre oferta de energía que hizo que estas especies acumularan grasa.

Estudios realizados para evaluar el crecimiento de otro tipo de bagres en jaulas flotantes como el chivo cabezón Ariopsis sp. (Castillo et al., 2006), muestran crecimientos similares a los encontrados en esta especie, a diferencia de otras como el pacú Piaractus mesopotamicus (Wicki y Luchini, 2005) que en tan solo unas semanas pueden alcanzar tamaños superiores a los encontrados en este estudio, lo que posiblemente indicaría que para estos bagres la ganancias en biomasa no son tan significativas como en otras especies.

Si se comparan los resultados obtenidos en esta investigación con los reportados a nivel comercial en el cultivo de especies exóticas como la trucha arco iris Oncorhynchus mykis (Morales y Quirós, 2007) y la tilapia Oreochromis niloticus (Llanes y Toledo, 2011), los parámetros zootécnicos obtenidos en este estudio pueden mostrar la potencialidad de esta especie para ser cultivada comercialmente teniendo en cuenta su rentabilidad, debido a que el capaz en comparación con las especies exóticas posee mayores precios de mercado (Sipsa, 2015). Sin embargo, es necesario realizar un análisis económico para determinar los costos de producción de esta especie en este tipo de sistemas.

En conclusión, el cultivo del capaz Pimelodus grosskopfii puede realizarse en jaulas flotantes bajo las condiciones de este estudio, como una alternativa para la diversificación de la acuicultura en el departamento de Huila.

\section{AGRADECIMIENTOS}

Los autores agradecen a Colciencias (Convenio No. 1572013) por el apoyo financiero, al Centro de Desarrollo Tecnológico Piscícola Surcolombiano-ACUAPEZ, Universidad Surcolombiana, Autoridad Nacional de Pesca y Acuicultura-AUNAP y estación piscícola Comepez por el apoyo logístico.

\section{BIBLIOGRAFÍA}

Amisah, H., Oteng, M.A. y Ofori, J.K. 2009. Growth performance of the African catfish, Clarias gariepinus, fed varying inclusion levels of Leucaena leucocephala leaf meal. Journal of applied Sciences and environmental Management 13(1): 21-26.

Arce, E. y Luna-Figueroa, J. 2003. Efecto de dietas con diferente contenido proteico en las tasas de crecimiento de crías del Bagre del Balsas Ictalurus balsanus (Pisces: Ictaluridae) en condiciones de cautiverio. Aquatic 18: 39-47.

Barcellos, L.J., Kreutz, L.C., Mezzalira-Quevedo, R., Fioreze, I., Cericato, L., Benck-Soso, A., Fagundes, M., Conradd, J., Krammer-Baldissera, R., Bruschi, A. y Ritter, F. 2004. Nursery rearing of jundia, Rhamdia quelen (Quoy y Gaimard) in cages: cage type, stocking density and stress response to confinement. Aquaculture 232: 383-394.

Castillo, L., Dávila, J., Chaparro, N., Hernández, A., Gaitán, S. y Acero, A. 2006. Engorde en jaulas del bagre estuarino "chivo cabezón" Ariopsis sp. (Miles, 1945) en la Ciénaga grande de Santa Marta, Colombia. Intrópica 3: 59-67.

Cala, P., Pérez, C. y Rodríguez, I. 1996. Aspectos Bioecológicos de la población de capaz, Pimelodus grosskopfii, (Pisces: Pimelodae) en el embalse de Betania y parte alta del río Magdalena, Colombia. Revista Academia Colombiana de Ciencias Exactas 20(77): 319-330. 
Cala, P. 1997. Espermatogénesis y ciclo anual reproductivo del capaz Pimelodus grosskopfii, (Pisces: Pimelodae) en el alto río Magdalena, Colombia. Caldasia 19(1): 45-53.

Cho, S.H. y Lovell, R.T. 2001. Variable feed allowance with constant protein input for channel catfish Ictalurus punctatus cultured in ponds. Aquaculture 204: 101-112.

Coldebella, I.J., RadünzNetom, J., Mallmann, C.A., Veivergerg, C.A., Bergamin, G.T., Pedron, F.A., Ferreira, D. y Barcellos, L.J. 2012. The effects of different protein levels in the diet on reproductive indexes of Rhamdia quelen females. Aquaculture 312(4): 137-144.

Cruz-Casallas, N.E., Marciales-Caro, L.J., Díaz-Olarte, J.J., Murillo-Pacheco, R., Medina-Robles, V. y Cruz-Casallas, P.E. 2010. Desempeño productivo del yaque (Leiarius marmoratus Gill, 1870) bajo diferentes densidades de siembra en estanques en tierra. Revista Colombiana de Ciencias Pecuarias 23: 22-28.

Esquivel, B.M., Esquivel, J.R. y Zaniboni, F.E. 2008. Effects of stocking density growth Catfish Ictalurus punctatus, Fingerlings in Southern Brazil. Journal of applied Aquaculture 7: 1-6.

Fagbenro, O.A. y Arowosoge, I.A. 1991. Growth response and nutrient digestability by Clarias isherienses (Sydenham, 1980) fed varying levels of dietary coffee pulp as replacement for maize in low-cost diets. Bioresource Technology 37: 253-258.

Froese, R. 2006. Cube law, condition factor and weight-length relationships: history, meta-analysis and recommendations. Journal of Applied Ichthyology 22:241-253.

González, P. y Oyarzún, C. 2002. Variabilidad de índices biológicos en Pinguipes chilensis Valenciennes 1833 (Perciformes, Pinguipedidae): ¿Están realmente correlacionados? Gayana 66:249-253.

Kennedy-Luz, R. y Zaniboni-Filho, E. 2002. Larvicultura do Mandi-amarelo Pimelodus maculatus Lacépède, 1803 (Siluriformes: Pimelodidae) em Diferentes Densidades de Estocagem nos Primeiros Dias de Vida. Revista Brasileira de Zooctenia 1(2): 560-565.

Linhart, O., Stech, L., Svarc, J., Rodina, M., Audebert, J.P., 26 Grecu, J. y Billard, R. 2002. The culture of the European catfish, Silurus glanis, in the Czech Republic and France. Aquatic Living Resources 15:139-144.

Llanes, J. y Toledo, J. 2011. Desempeño productivo de la tilapia del Nilo (Oreochromis niloticus) con la inclusión de altos niveles de harina de soya en la dieta. Revista Cubana de Ciencia Agrícola 45(2): 183-186.

Mancera-Rodríguez, N.J. y Cala, P. 1997. Aspectos bioecológicos de la comunidad íctica asociada a un cultivo de tilapia roja en jaulas flotantes en el embalse de Betania, Colombia. Dhalia 2:31-53.

Mojica, H.O., Rodríguez, J.A. y Orozco, C.R. 2003. Manual de reproducción y cultivo: El bagre rayado (Pseudoplatystoma fasciatum). Instituto Nacional de pesca y acuicultura INPA, Villavicencio.

Morales, G.A. y Quiroz, R. 2007. Desempeño productivo de la trucha arco iris en jaulas bajo diferentes estrategias de alimentación. Asociación Latinoamericana de producción animal 15(4): 119-127.

Murillo-Pacheco, R., Cruz-Casallas, N.E., Ramírez-Merlano, J.A., Marciales-Caro, L.J., Medina-Robles, V.M. y CruzCasallas, P.E. 2012. Efecto del nivel de proteína sobre el crecimiento del yaque Leiarius marmoratus (Gill, 1870) bajo condiciones de cultivo. Orinoquia 16(2):52-61.

Namrata, S. y Shamrao, S. 2013. Studies on Condition Factor of Catfish Macrones vittatus (Bloch, 1794) from Bhategaon Dam, District Hingoli (M. S.). Zoology 2(9): 440-442.

Núñez, J., Dugué, R., Corcuy-Arana, N., Duponchelle, F., Renno, J.F., Raynaud, T., Hubert, N. y Legendre, M. 2008. Induced breeding and larval rearing of Surubí, Pseudoplatystoma fasciatum (Linnaeus, 1766), from the Bolivian Amazon. Aquaculture Research 39: 764-776.

Petrere, M., Borges, R., Agudelo, E. y Corrales, B. 2004. Review of the large catfish fisheries in the upper Amazon and stock depletion of piraiba (Brachyplatystoma filamentosum Lichtenstein). Review and Fish Biology and Fisheries 14: 403-414.

Phillips, A.T., Sumerface, C.R. y Clayton, A.R. 1998. Feeding frequency effects on water quality and growth of Walleye fingerlings in intensive culture. The Progressive Fish Culturist 60(1):1-8.

Román-Valencia, C. 2001. Ecología trófica y reproductiva de Trichomycterus caliense y Astroblepus cyclopus (Pisces: Siluriformes) en el río Quindío, Alto Cauca, Colombia. Revista Biológica Tropical 49(2): 657-666.

Rowland, S.T., Mifsud, C., Nixon, M. y Boyd, P. 2006. Effects of stocking density on the performance of the Australian freshwater silver perch (Bidyanus bidyanus) in cages. Aquaculture 253: 301-308. 
Segura, L., Hayashi, C., De Souza, S. y Soares, C. 2004. Canibalismo entre larvas de pintado, Pseudoplatystoma corruscans, cultivadas sobre diferentes densidades de estocagem. Acta Scientiarum Biological Sciences 26(3): 299-302.

Sena de Souza, L., Osório, J.L., Fernandes, P., Ortiz, S. y Dos Santos Vaz, B. 2005. Crescimento e sobrevivência do catfi sh de canal (Ictalurus punctatus) e jundiá (Rhamdia sp.) no outono-invernodo Rio Grande do Sul. Ciência Rural 35:891-896.

Sipsa. 2015. Sistema de información de precios y abastecimiento del sector agropecuario._URL:_https:// www.dane.gov.co/files/investigaciones/agropecuario/ sipsa/Sem 03ene_09ene_2015.pdf. Consultado: 20 de abril del 2015.

Valbuena-Villarreal, R.D., Zapata-Berruecos, B. y CruzCasallas, P.E. 2010. Reproducción inducida de Capaz (Pimelodus grosskopfii) con extracto de hipófisis de carpa: reporte preliminar. Orinoquia 14(2): 133-139.

Valbuena-Villarreal, R.D., Zapata-Berruecos, B., DavidRuales, C. y Cruz-Casallas, P.E. 2012a. Desarrollo Embrionario del Capaz Pimelodus grosskopfii (Steindachner, 1879). International Journal of Morphology 30(1): 150-156.
Valbuena-Villarreal, R.D., Zapata-Berruecos, B. y GutiérrezEspinoza, M.C. 2012b. Coeficientes de digestibilidad aparente de tres ingredientes proteicos para capaz, Pimelodus grosskopfii. Orinoquia 16: 179-186.

Valbuena-Villarreal, R.D., Zapata-Berruecos, B. y OteroPaternina, A. 2013. Evaluación de la primera alimentación en larvas de capaz Pimelodus grosskopfii bajo condiciones de laboratorio. Revista MVZ Córdoba 18(2): 3518-3524.

Villa-Navarro, F.A. y Losada-Prada, S. 1999. Hábitos alimenticios de Pimelodus grosskopffi y Ageneiosus caucanus (Pisces:Siluriformes) en la represa de Prado (Tolima). pp 224. En: XXXIV Congreso Nacional de Ciencias Biológicas, Cali.

Villa-Navarro, F.A. 2002. Diferenciación entre poblaciones de Pimelodus clarias y Pimelodus grosskopffi (Siluriformes: Pimelodidae) en la cuenca del río Magdalena Colombia. Tesis de Maestría, Universidad del Valle, Cali, Colombia.

Wicki, G. y Luchini, L. 2005. Ensayo de engorde del Pacu (Piaractus mesopotamicus, Holmberg, 1887) en jaulas. Natura Neotropicalis 36: 45-50.

Fecha de recepción: 26/05/2015

Fecha de aceptación: 29/07/2015

Para citar este artículo: Carrera, S y R.D. Valbuena-Villareal. 2015. Desempeño productivo del Capaz (Pimelodus grosskopfii, Steindachener, 1879) bajo diferentes densidades de siembra y contenido de proteína en la dieta. Revista Intropica Vol. 10: 20 - 27 